\title{
Quantum effects in band gap-modulated amorphous carbon superlattices
}

\author{
V Stolojan ${ }^{1}$, P Moreau $^{2}$, M J Goringe ${ }^{3}$ and S Ravi P Silva ${ }^{1}$ \\ ${ }^{1}$ Advanced Technology Institute, University of Surrey, Guildford, GU2 7XH, UK \\ 2 Institut des Matériaux Jean Rouxel, Université de Nantes-CNRS, Laboratoire de Chimie des Solides, \\ 44322 Nantes, France \\ ${ }^{3}$ School of Engineering, University of Surrey, Guildford, GU2 7XH, UK
}

\begin{abstract}
Diamond-like carbon (DLC) films, with their controllable optical band gaps from 1$4 \mathrm{eV}$, have promised much as electronic semiconducting materials for many years. Particularly, hydrogenated amorphous carbon films $(\mathrm{a}-\mathrm{C}: \mathrm{H})$ have attracted interest in their possible use in light emitting diodes, flat panel displays and solar cells. Photoemission in these films can be explained by the electron-hole recombination at $\mathrm{sp}^{2}$-bonded carbon clusters, enhanced by the quantumconfinement of the rigid $\mathrm{sp}^{3}$-matrix. The speed of such devices can be improved by constructing homogenous carbon superlattices from alternate high and low band-gap a-C:H layers. Here, we measure directly the electronic properties across superlattices, using Energy Loss Spectroscopic Profiling (ELSP) in a Transmission Electron Microscope (TEM). By analysing the valence losses across the layers, we characterise the electronic structure of the films. By modelling the influence of the interface collective modes of oscillation (interface plasmons), we show that the measured changes in the bulk plasmon energies of the wells are due to quantum confinement and predicted using the 'particle-in-a-box' model. These a-C superlattice structures have the potential of introducing a highly versatile novel large area amorphous semiconductor that is carbon based and deposited at room temperature.
\end{abstract}

\section{INTRODUCTION}

Band-gap modulated artificial structures tailor the energy levels by quantizing the electron wavefunction's momentum and energy. They have been introduced as a practical solution to the demand for increasing speeds of operation, beyond the fundamental limits available with atomic energy leves. The resulting quantized energy levels in these artificial superlattices are controlled by the well's width and depth. These energy levels can also degenerate into bands, when tunnelling is controlled through the height and widths of the barriers separating the wells. This can lead to the tailoring of devices for specific applications, such as high-frequency generators for the mobile phone industry.

\section{EXPERIMENTAL METHOD}

\subsection{Energy Loss Spectroscopic Profiling (ELSP)}

A Gatan Imaging Filter (GIF) attached to a transmission electron microscope (TEM) provides a three-dimensional data set (3D) which can be recorded using a two-dimensional detector (the chargecoupled-device CCD). The three dimensions available are the two dimensions of the magnified projected image of the sample and the one energy loss dimension. An energy filtered image is essentially a slice, at constant energy, through this 3D data set (Fig. 1a). An energy loss spectrum requires the integration of the two spatial dimensions, and this is done by the quadrupole lenses of the GIF in one of the spatial dimensions and by binning in the CCD over the other dimension. However, 
we can see that, before binning, the CCD actually records a 2D image with one spatial axis and one energy loss axis orthogonal to the spatial axis (Fig. 1b). ELSP is applicable to linear features, such as

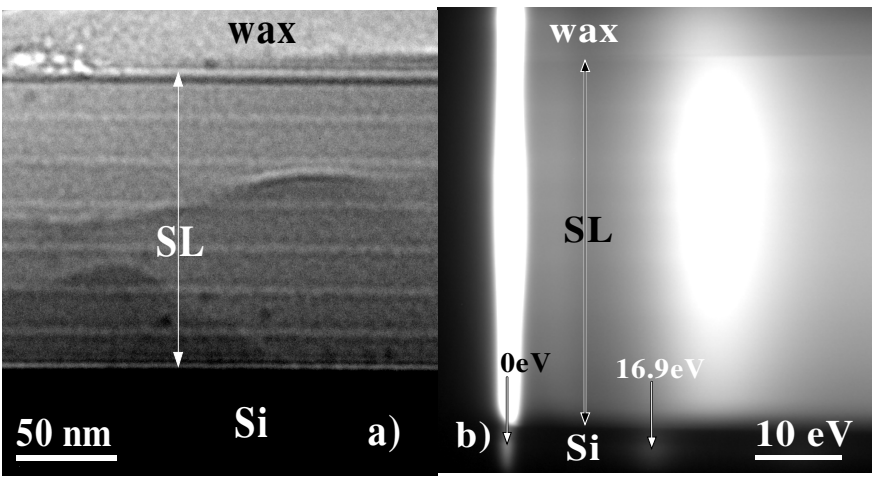

Figure 1 a) Energy filtered image of a superlattice at $5 \mu \mathrm{m}$ defocus, showing the wells with bright contrast. b) The corresponding low loss ELSP image; the vertical axis is identical, to within a scaling factor, to Fig. 1a. planar interfaces, when they can be rotated so that they lie parallel to the spatial axis integrated by the quadrupoles (Walther 2003). The image recorded at the CCD is then a set of spectra where each one corresponds to a linear section of the sample, parallel to the interface, taken at positions across the interface. The number of positions (and hence the sampling of the spatial dimension) is controlled by the dispersion provided by the quadrupoles, the chromatic aberration, sample drift and, if several images are averaged for improving the signal-to-noise ratio, by the ability to align accurately ELSP images with respect to each other.

\subsection{Sample Preparation and Characteristics}

\begin{tabular}{|c|c|c|}
\hline Sample & Well & Barrier \\
\hline $\begin{array}{c}\text { Optical } \\
\text { bandgap }\end{array}$ & $1.5 \mathrm{eV}$ & $2.8 \mathrm{eV}$ \\
\hline $\begin{array}{c}\text { Refractive } \\
\text { index }\end{array}$ & $\mathbf{2 . 2}$ & $\mathbf{2 . 1}$ \\
\hline $\begin{array}{c}\text { H } \\
\text { concentration }\end{array}$ & $\mathbf{3 6 \%}$ & $\mathbf{3 3 \%}$ \\
\hline $\mathrm{Sp}^{2}$ & $\mathbf{8 7 \%}$ & $\mathbf{7 4 \%}$ \\
\hline
\end{tabular}

Table 1 Properties of test films deposited under identical conditions as the barriers and wells of the superlattices (Silva 1994a, Davis 1995).
The homogenous superlattices were deposited on $\mathrm{Si}(100)$ substrates using plasma-enhanced chemical vapour deposition, by alternating the biasing voltage between 190V (for wells) and -265V (for barriers), under computer control (Silva 1994a). Cross-sectional TEM samples were prepared by mechanical grinding and polishing, followed by ion-beam polishing. Test barrier and well films were deposited under identical conditions, and were etched away from the Si substrates using a $\mathrm{HF}: \mathrm{HNO}_{3}$ acid mix. Samples were analysed in a Philips CM200 (200kV, LaB 6 ) TEM fitted with a GIF2000 spectrometer. The average energy dispersion and spatial dispersion used for ELSP were 0.1 eV/pixel and $0.45 \mathrm{~nm} /$ pixel (calibrations were performed for each individual experiment). Table 1 summarises some of the properties of the barriers and wells (Davis 1995).

\subsection{Data Processing}

For each superlattice, we collected 40 ELSP images containing the valence loss across the superlattices, 40 ELSPs with the beam going through the vacuum and another 40 with the beam through the vacuum and a preset voltage wobble on the drift tube, for energy calibration purposes. Each ELSP set was aligned in energy to within a pixel and the valence loss set was also aligned in the spatial dimension on either the superlattice/Si interface or the superlattice/mounting wax interface (or both) to within a pixel. The spatial alignment parameters thus gathered are good measure of the sample drift within one acquisition period ( $0.5 \mathrm{~s}$ for all ELSP images). The final images contained were $\sim 600 \times 600$ pixels, i.e. 600 spectra sampling the dimension perpendicular to the interface with $\sim 0.45$ $\mathrm{nm} /$ pixel. For each spectrum, the origin of the energy loss scale was determined to within $20 \mathrm{meV}$ using a Lorentzian fit to the zero-loss peak and similarly the energy dispersion was determined from the calibration ELSP. The plasmon energy was determined using a Lorentzian curve to within $10 \mathrm{meV}$.

\section{SPATIAL RESOLUTION}

The issue of spatial resolution can be resolved into two distinct items: one relates to the experimental set-up (chromatic aberration, sample drift, data processing) and the other relates to the 
spatial extent of the energy loss event studied.

The experimental spatial resolution in this case is mostly affected by the ability to align the ELSP images to within a pixel; for a normal distribution of positions about the centre, this leads to a broadening of $\sim 0.95 \mathrm{~nm}$. The short exposure times used lead to a 0.1 pixel broadening. The influence of chromatic aberration is negligible due to the small range of the energy loss of interest.

The event delocalisation for collective excitations (plasmons) is quite broad, up to several nanometres (Rivacoba 2000). However, at the interfaces between two dielectrics, interface plasmons can be established. These surface states, much more confined normal to the interface than bulk plasmons, act to screen out the contribution of the bulk plasmons to the scattering cross-section. This is known as the 'begrezungs' effect and manifests mathematically as a subtraction of the bulk contribution from the total scattering cross section, as one approaches the interface (Howie 1983). This means that the final result is not a superposition of the surface excitation to the bulk excitation but a replacement. We modelled the energy loss of the relativstic electron using the classical dielectric approach (Garcia-Molina 1985) for a sandwich interface (Turowski 1994, Moreau 1997), using dedicated software (Walsh 1992). This was done using the dielectric functions extracted from the energy loss spectra of barrier and well test layers grown under identical conditions as the superlattices. Figure 2a shows the change in the plasmon energy of the well as a function of well width, indicating that the plasmon energy of the well is defined down to well widths of $\sim 1 \mathrm{~nm}$, beyond which the surface plasmons couple and the plasmon energy measured, is no longer representative of the well. Figure $2 \mathrm{~b}$ models the variation of the plasmon energy across a $3 \mathrm{~nm}$ wide well, corrected for the experimental broadening of $1 \mathrm{~nm}$. The $\sim 1 \div 1.5 \mathrm{~nm}$ region over which the plasmon energy changes from its barrier to its well value is an overall measure of the spatial resolution of this method when using valence losses, applied to sandwich structures.
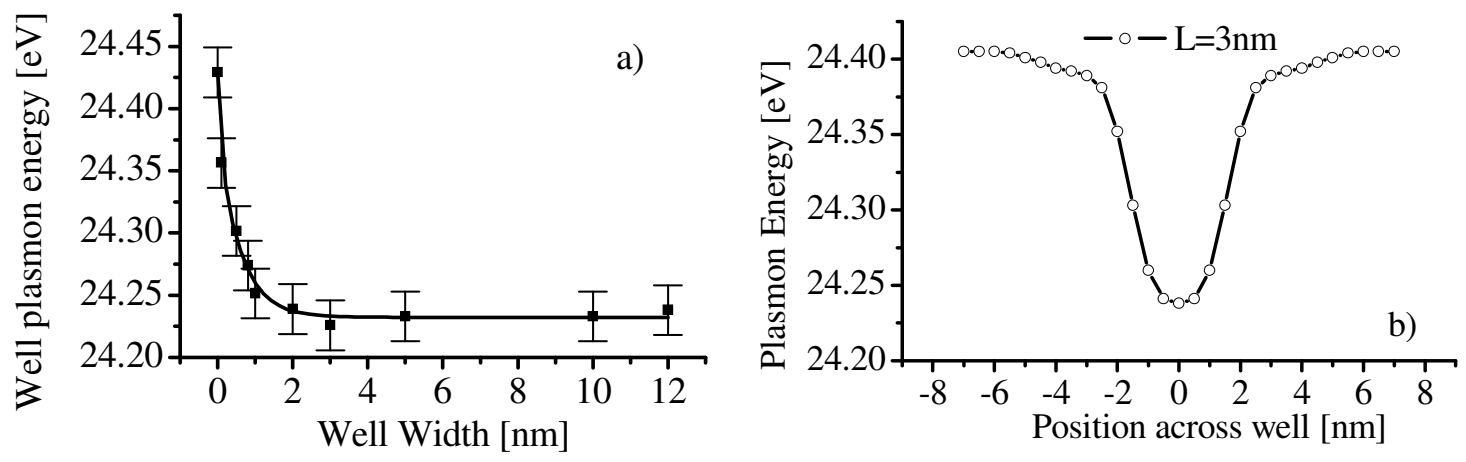

Figure 2 a) variation of the plasmon energy in the middle of the well, as a function of well width. The line fit is a guide to the eye. b) variation of the plasmon energy across a $3 \mathrm{~nm}$ wide well, corrected for the $1 \mathrm{~nm}$ experimental broadening.

\section{RESULTS}

The plasmon energy $E_{\mathrm{P}}$ for electrons bound by a bandgap $\mathrm{E}_{\mathrm{G}}$ can be expressed as:

$$
\mathrm{E}_{\mathrm{p}}^{2}=\mathrm{E}_{0}^{2}+\mathrm{E}_{\mathrm{G}}^{2}
$$

where $\mathrm{E}_{0}^{2}=\hbar^{2} \mathrm{ne}^{2} / \mathrm{m} \varepsilon_{0}$ is the free electron plasmon energy (Egerton 1996). Figure 3a shows a typical profile of the plasmon energy across a superlattice. The gap energy here is the Penn gap, a parameter modified from the optical band gap to account for scattering of electrons at Brillouin zone boundaries and non-dipole transitions. The Penn gaps are calculated (Penn 1962) as $\mathrm{E}_{\text {gbarrier }}=13.210 \mathrm{eV}$ and $\mathrm{E}_{\mathrm{gwell}}=12.36 \mathrm{eV}$. Assuming that the free electron values do not change with well widths, Fig. $3 \mathrm{~b}$ shows the experimental change in the Penn gap of the wells, as a function of well widths. This can be modelled using the 'particle-in-a-box', where the well depth is $0.425 \mathrm{eV}$ (half of the Penn bandgap difference). By matching the wavefunction at the boundary, we calculate the expected change in the Penn bandgap due to quantum confinement within a well of changing width, using the effective mass as a parameter (Silva 1994a). 

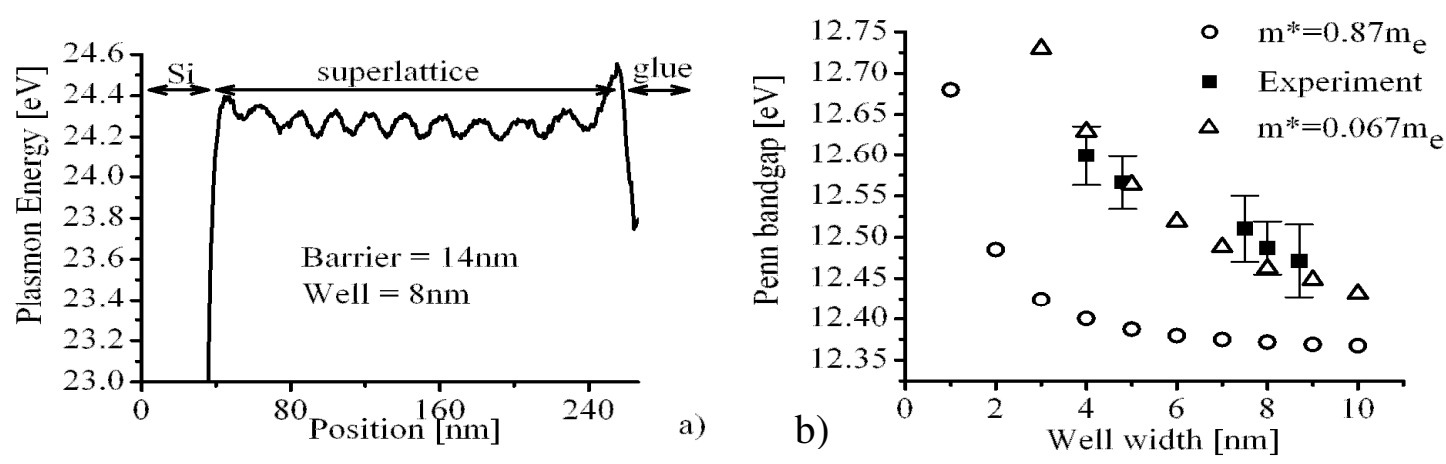

Figure 3 a) The variation of the plasmon energy across a typical superlattice. b) The measured Penn bandgap energies compared with the predicted changes in bandgap energy, for $\mathrm{m}^{*}=0.067 \mathrm{~m}_{\mathrm{e}}$ (open triangles) and for $\mathrm{m}^{*}=0.87 \mathrm{~m}_{\mathrm{e}}$ (open circles).

There are two values of the effective mass available in literature, which are applicable here. The first, $\mathrm{m}^{*}=0.87 \mathrm{~m}_{\mathrm{e}}$ has been used to describe the relationship between the density of valence electrons and the plasmon energy for a range of amorphous carbons (Ferrari 2000), whilst the second, $\mathrm{m}^{*}=0.067 \mathrm{~m}_{\mathrm{e}}$ has been used to describe the measured change in the optical bandgaps of the superlattices studied here, as a function of well width (Silva 1994b). Figure 3b shows the theoretical predictions for each of the two effective masses, and we can see that $\mathrm{m}^{*}=0.067 \mathrm{~m}_{\mathrm{e}}$ is a good fit of the experimental data. This value of the effective mass is a consequence of the 'particle-in-a-box' model used and can be related to the large length of the barriers and consequently the very small amount of tunnelling in this case.

\section{CONCLUSION}

We have confirmed that effects associated to quantum confinement are possible in amorphous carbon semiconductor architectures and that these can be investigated using valence losses to a spatial resolution approaching $1 \mathrm{~nm}$. This is because of the screening provided by the surface plasmons established at the interfaces between the barriers and wells of the superlattices.

\section{ACKNOWLEDGEMENTS}

We are very grateful to the EPSRC for funding through the Portfolio Partnership Grant.

\section{REFERENCES}

Davis C A et al 1995 Phys. Rev. Lett. 75, 4258

Egerton R F 1996 EELS in the Electron Microscope $2^{\text {nd }}$ Ed. (New York London - Plenum Press) ch. 3 Ferrari A C et al. 2000 Phys. Rev. B62, 11089

Garcia Molina R, Gras Marti A, Howie A and Ritchie R H 1985 J. Phys. C18, 5335

Howie A 1983 Ultramicroscopy 11, 141

Moreau P, Brun N, Walsh C A, Colliex C and Howie A 1997 Phys. Rev. B56, 6774

Penn D R 1962 Phys. Rev. 128, 2093

Rivacoba A, Zabala N and Aizpurua J 2000 Prog. Surf. Sci. 65, 1

Silva S Ravi P et al. 1994a Thin Sol. Films 253, 20

Silva S Ravi P et al. 1994b Jpn. J. Appl. Phys. 33, 6458

Turowski M A, Kelly T F and Batson P E 1994 J. Appl. Phys 76, 3776

Walsh C A 1992 Computer Programmes for the Calculation of Electron Energy-Loss Spectra from Interfaces Between Dielectric Media (Cavendish Laboratory, Cambridge)

Walther T 2003 Ultramicroscopy 96, 401 